\title{
Plasma C-reactive protein is not related to sinus non-conversion by maze procedure adjunct to mitral valve surgery
}

\author{
Chien-Jen Chen ${ }^{\mathrm{a}, 1}$, Wen-Hao Liu ${ }^{\mathrm{a}, 1}$, Jen-Ping Chang ${ }^{\mathrm{b}}$, Wan-Chun $\mathrm{Ho}^{\mathrm{a}}$, Yung-Lung Chen ${ }^{\mathrm{a}}$, \\ Chih-Yuan Fang ${ }^{\mathrm{a}}$ and Mien-Cheng Chen ${ }^{\mathrm{a}, *}$ \\ ${ }^{a}$ Division of Cardiology and Department of Internal Medicine, Kaohsiung, Taiwan, China \\ ${ }^{\mathrm{b}}$ Division of Cardiovascular Surgery, Kaohsiung Chang Gung Memorial Hospital and Chang Gung University \\ College of Medicine, Kaohsiung, Taiwan, China
}

\begin{abstract}
In Framingham cohort study, C-reactive protein was not associated with incident atrial fibrillation (AF) after adjustment for left atrial size. This study examined whether levels of plasma inflammatory markers would be significant risk factors for failed maze procedure for AF. This study enrolled 88 patients with mitral valve disease undergoing valve surgery $(n=32$, sinus control group) or concomitant maze procedure for persistent atrial fibrillation (AF) ( $n=56$, AF group). The mean follow-up in the AF group was $55.0 \pm 17.5$ months. The AF and sinus control groups did not differ in preoperative levels of C-reactive protein $(p=0.636)$. In the AF group receiving maze procedure, the sinus conversion $(n=37)$ and non-conversion $(n=19)$ groups did not significantly differ in preoperative levels of interleukin-6 $(p=0.607)$ and tumor necrosis factor- $\alpha(p=0.379)$. In multivariate analysis after adjustment for preoperative plasma inflammatory markers, independent factors associated with sinus conversion were AF duration ( $p=0.003)$, and left atrial area $(p=0.014)$. In conclusion, plasma inflammatory markers are not associated with sinus non-conversion by radiofrequency maze procedure.
\end{abstract}

Keywords: Atrial fibrillation, inflammation, maze procedure, mitral valve surgery

\section{Introduction}

Atrial fibrillation (AF), the most frequent sustained cardiac arrhythmia, has been shown to increase the risk of systemic embolization and mortality [1,2], and is frequently observed in clinical situations associated with atrial enlargement, such as mitral valve disease. Mitral valve disease is among the more important underlying causes of heart failure [3-5]. We have shown that preoperative and postoperative atrial sizes and AF duration

\footnotetext{
* Corresponding author: Mien-Cheng Chen, MD, Division of Cardiology, Department of Internal Medicine, Kaohsiung Chang Gung Memorial Hospital, 123 Ta Pei Road, Niao Sung District, Kaohsiung City, 83301, Taiwan, China. Tel.: +886 77317123 ext. 8300; Fax: +886 7732 2402; E-mail: chenmien@ms76.hinet.net.

${ }^{1}$ Chien-Jen Chen, MD and Wen-Hao Liu, MD contributed equally to this article.
}

are primary predictors of sinus conversion by radiofrequency maze procedure for patients with persistent AF and mitral valve disease [6,7].

Epidemiological and clinical studies have identified an association between C-reactive protein (CRP) and both the presence of $\mathrm{AF}$ and risk of developing future AF [8]. Cytokines [tumor necrosis factor- $\alpha$ (TNF- $\alpha$ ), interleukin- $1 \beta$ and particularly, interleukin-6 (IL-6)] stimulate and induce hepatic production of other acutephase reactants, such as CRP. However, CRP, an acutephase protein and a marker of systemic inflammation, is produced in non-specific physiological and biochemical responses to most forms of organ or tissue damage, infection and inflammation [9]. Roldan et al. showed that high plasma levels of interleukin-6 in AF appear to be related to clinical variables in non-rheumatic AF patients rather than to the presence of AF per se [10] 
Additionally Akar et al. showed no association between acute onset human AF and plasma inflammatory markers [11]. In Framingham cohort study, CRP was not associated with incident AF after adjustment for left atrial size [12]. Accordingly, this study investigated the association between plasma inflammatory markers and the development of AF after radiofrequency maze procedure in patients undergoing concomitant mitral valve surgery.

\section{Methods}

\subsection{Patient population}

This study conducted a case-control cohort analysis from 233 patients with mitral valve disease undergoing valve surgery and concomitant radiofrequency maze procedure for persistent $\mathrm{AF}(n=189)$ or valve surgery alone (sinus control; $n=44$ ). Exclusion factors include febrile disorder, hyperthyroidism, infectious or inflammatory disease, autoimmune disease, malignancy, chronic renal failure (serum creatinine $>$ $2.5 \mathrm{mg} / \mathrm{dL}$ ), acute or chronic viral hepatitis and use of immunosuppressive drugs. An estimated sample size of 60 patients ( $n=30$ for each group) was based on the effective size with an $\alpha=0.05$, a power of $80 \%$, using a CRP interquartile range of 0.63 to $3.4 \mathrm{mg} / \mathrm{L}$ during sinus rhythm in paroxysmal AF patients, according a previous study [13]. A case-control sample was randomly selected of those who had plasma frozen and stored at baseline before surgery and during follow-up ( $n=88$ ). The study cases (AF group; $n=56$ ) comprised subjects with persistent AF [mean $( \pm \mathrm{SD})$ with duration of $54.1 \pm 60.8$ months; duration range, 1 to 183 months] before surgery. All patients in the AF group had mitral valve disease, $39(69.6 \%)$ patients suffered concomitant tricuspid valve disease and seven $(12.5 \%)$ patients suffered concomitant aortic valve disease. The radiofrequency maze procedure was performed simultaneously with the following procedures: mitral valve replacement (mechanical valve, $n=8$; tissue valve, $n=18$ ), mitral valve repair $(n=30)$, aortic valve replacement (mechanical valve, $n=2$; tissue valve, $n=2$ ), aortic valve repair $(n=3)$, tricuspid tissue valve replacement (mechanical valve, $n=1$; tissue valve, $n=1$ ), and tricuspid repair or annuloplasty $(n=37)$. Thirty-two age- and sex-matched controls with sinus rhythm (sinus control group) were also randomly sampled and biochemical analysis was performed. All patients in the sinus group had mitral valve disease, eight $(25.0 \%)$ patients had concomitant tricuspid valve disease and five $(15.6 \%)$ patients had concomitant aortic valve disease. The baseline and follow-up data were prospectively obtained. Informed consent was obtained from all subjects. The study protocol was approved by the Institutional Review Committee for Human Research at our institution.

\subsection{Radiofrequency maze procedure}

All the AF patients enrolled in this study received the same maze procedure. The maze procedure was performed with handmade saline solution-irrigated, radiofrequency catheter ablation guided by direct visualization, which has been described in details by Chen et al. [14]. In brief, the radiofrequency ablation part of the surgical procedure replaced most of the atrial incisions used in the Cox maze III procedure, with the exception of an incision in the right atrial free wall and a standard left atrial incision in the Waterston groove, which were used to enter both atrial cavities. The left atrial appendage was ligated, and a cryoablation lesion (15-mm head probe at $-60^{\circ} \mathrm{C}$ for $2 \mathrm{~min}$; Frigitronics; Shelton, CT) was created around the orifice. The right and left islands of the pulmonary veins were isolated separately and then interconnected with an additional line in the inferior left atrial floor. Cryoablation was also applied to produce lesions in the proximal coronary sinus and away from the posterior descending artery. The right atrial maze procedure was performed as follows: the right atrium was opened through the excised right atrial appendage; and an anterior incision was made from the middle of the anterolateral aspect of the base of the amputated auricle toward the inferior caval vein orifice. Additional radiofrequency ablation lines were drawn from the medial aspect of the base of the excised right atrial appendage into the annulus of the tricuspid valve and from the caudal end of the first radiofrequency ablation line at the atrioventricular groove to the posterior part of the annulus of the tricuspid valve. The septal stage of the procedure was performed as the Cox maze III procedure, but the intercaval counterablation was moved posterolaterally.

\subsection{Echocardiography}

Transthoracic echocardiographic examinations were performed on all patients on the day before valve surgery and at 3-month follow-up after surgery [6,7]. 


\subsection{Blood sample collection and measurement of plasma high-sensitivity $C$-reactive protein, tumor necrosis factor- $\alpha$ and interleukin- 6 concentrations}

Blood samples from all study subjects were obtained immediately before valve surgery during the fasting period, non-sedative state on study entry and at 3-month follow-up. The plasma was immediately separated and frozen at $-80^{\circ} \mathrm{C}$ until the assay. The human plasma high-sensitivity CRP (hs-CRP) (AssayPro; Missouri, USA), high-sensitivity TNF- $\alpha$ (R and D Systems; Minnesota, USA) and high-sensitivity IL-6 concentrations ( $\mathrm{R}$ and D Systems; Minnesota, USA) were quantified using a commercially available ELISA. The samples were processed according to the manufacturer's instructions.

\subsection{Electrocardiography and medications at follow-up}

After hospital discharge, patients underwent monthly follow-up for cardiac rhythm assessment by 12lead surface electrocardiograms for six months and thereafter, electrocardiograms were obtained every 23 months on clinic visit, or if cardiac symptoms developed. No class I or III anti-arrhythmic agents were prescribed to patients at follow-up.

\subsection{Statistical analysis}

Data are presented as means $\pm \mathrm{SD}$ or percentages. Categorical variables were compared using the chisquare or Fisher exact tests, as appropriate. Moreover, continuous variables were compared by the MannWhitney U test. The significance of multiple variables identified as significant via univariate analysis was calculated using forward stepwise logistic regression analysis. To compare the predictive values of significant variables in logistic regression analysis, areas under the Receiver Operator Characteristic curve were constructed for sensitivity and specificity to predict AF development after the maze procedure. The best bound for predicting AF development was defined as that producing the highest sensitivity and specificity for differentiating sinus conversion and sinus non-conversion patients. In risk stratification analysis of event rates of AF during follow-up after radiofrequency maze procedure, we divided AF patients into four groups on the basis of whether they were above or below the threshold of the AF duration and left atrial area according to the predic- tor cutoff values determined by the discriminant analysis. Differences between groups were compared by means of the log-rank test, and Kaplan-Meier survival curves were generated for each group. Finally, statistical analyses were performed using a statistical software program (SPSS version 13.0; SPSS Inc.; Chicago, Illinois, U.S.A.). All $p$ values were two-tailed, and the level of statistical significance was set at $p<0.05$.

\section{Results}

\subsection{Baseline clinical characteristics, echocardiographic data and levels of inflammatory markers of both $A F$ and sinus control patients before surgery}

Table 1 lists the clinical characteristics of the study patients. The AF and sinus control groups did not significantly differ in age, gender, body mass index, heart failure status, leukocyte count, or prevalence of diabetes mellitus, hypertension and hyperlipidemia. However, the AF group contained significantly more patients with significant tricuspid regurgitation than the sinus control group. The AF and sinus control subgroups were balanced in terms of use of drugs such as statins, $\beta$-blockers and $\mathrm{Ca}$-channel blockers before surgery. However, the AF group contained significantly more patients taking digoxin and warfarin, and fewer patients taking angiotensin converting enzyme inhibitors or type I angiotensin II receptor blockers before surgery. Furthermore, the preoperative left atrial diameter, left atrial area and right atrial area were significantly larger in the AF group than the sinus control group.

The AF and sinus control groups did not differ significantly in preoperative levels of hs-CRP and IL-6 (Table 1).

\subsection{Echocardiographic data and levels of inflammatory markers of AF and sinus control patients after surgery}

Table 2 lists the echocardiographic data and levels of inflammatory markers of the study patients after surgery. The postoperative left atrial diameter, left atrial area and right atrial area at 3-month follow-up were significantly larger in the AF group than the sinus control group. The AF and sinus control groups did not differ significantly in levels of inflammatory markers after surgery. 
Table 1

Baseline clinical characteristics of patients studied before surgery

\begin{tabular}{|c|c|c|c|}
\hline & $\begin{array}{l}\text { Atrial fibrillation group } \\
(n=56)\end{array}$ & $\begin{array}{c}\text { Sinus control group } \\
(n=32)\end{array}$ & $p$ value \\
\hline Age (years) & $56 \pm 13$ & $52 \pm 15$ & 0.320 \\
\hline Men $(\%)$ & $26(46.4 \%)$ & $18(56.3 \%)$ & 0.375 \\
\hline Body mass index $\left(\mathrm{kg} / \mathrm{m}^{2}\right)$ & $24.0 \pm 3.7$ & $24.1 \pm 3.7$ & 0.825 \\
\hline Duration of AF (months) & $54.1 \pm 60.8$ & & \\
\hline New York Heart Association classification & $56(100.0 \%)$ & $32(100.0 \%)$ & 0.327 \\
\hline II & $9(16.1 \%)$ & $7(21.9 \%)$ & \\
\hline III & $35(62.5 \%)$ & $22(68.8 \%)$ & \\
\hline IV & $12(21.4 \%)$ & $3(9.4 \%)$ & \\
\hline Previous embolism (\%) & $3(5.4 \%)$ & $3(9.4 \%)$ & 0.664 \\
\hline Mitral valve disease (\%) & $56(100.0 \%)$ & $32(100.0 \%)$ & 1.000 \\
\hline Tricuspid regurgitation (\%) & $39(69.6 \%)$ & $8(25.0 \%)$ & $<0.001$ \\
\hline Aortic valve disease (\%) & $7(12.5 \%)$ & $5(15.6 \%)$ & 0.681 \\
\hline Hypertension (\%) & $19(33.9 \%)$ & $10(31.3 \%)$ & 0.797 \\
\hline Diabetes mellitus (\%) & $10(17.9 \%)$ & $7(21.9 \%)$ & 0.646 \\
\hline Hyperlipidemia (\%) & $11(19.6 \%)$ & $10(31.3 \%)$ & 0.219 \\
\hline Coronary artery disease $(\%)$ & $3(5.4 \%)$ & $3(9.4 \%)$ & 0.664 \\
\hline $\begin{array}{l}\text { Angiotensin converting enzyme inhibitor or type I angiotensin } \\
\text { II receptor blocker }(\%)\end{array}$ & $32(57.1 \%)$ & $29(90.6 \%)$ & 0.001 \\
\hline Statins $(\%)$ & $1(1.8 \%)$ & $3(9.4 \%)$ & 0.135 \\
\hline Fibrate (\%) & $1(1.8 \%)$ & $0(0.0 \%)$ & 1.000 \\
\hline Beta blockade (\%) & $21(37.5 \%)$ & $6(18.8 \%)$ & 0.067 \\
\hline Calcium blockade (\%) & $13(23.2 \%)$ & $7(21.9 \%)$ & 0.885 \\
\hline Digoxin $(\%)$ & $41(73.2 \%)$ & $10(31.3 \%)$ & $<0.001$ \\
\hline Antiplatelets (\%) & $10(17.9 \%)$ & $4(12.5 \%)$ & 0.563 \\
\hline Warfarin $(\%)$ & $35(62.5 \%)$ & $0(0.0 \%)$ & $<0.001$ \\
\hline Propafenone $(\%)$ & $1(1.8 \%)$ & $0(0.0 \%)$ & 1.000 \\
\hline Amiodarone (\%) & $12(21.4 \%)$ & $0(0.0 \%)$ & 0.074 \\
\hline WBC (cells/uL) & $6364.3 \pm 2021.6$ & $6703.1 \pm 2423.9$ & 0.630 \\
\hline Serum creatinine $(\mathrm{mg} / \mathrm{dL})$ & $1.23 \pm 2.91$ & $1.09 \pm 1.36$ & 0.783 \\
\hline Left atrial diameter $(\mathrm{mm})$ & $54.4 \pm 10.3$ & $42.6 \pm 7.7$ & $<0.001$ \\
\hline Left atrial area $\left(\mathrm{cm}^{2}\right)$ & $45.9 \pm 19.4$ & $30.3 \pm 9.0$ & $<0.001$ \\
\hline Right atrial area $\left(\mathrm{cm}^{2}\right)$ & $26.2 \pm 8.6$ & $17.6 \pm 5.9$ & $<0.001$ \\
\hline Left ventricular ejection fraction (\%) & $64.6 \pm 12.3$ & $69.9 \pm 12.6$ & 0.033 \\
\hline high-sensitivity C-reactive protein $(\mathrm{mg} / \mathrm{L})$ & $1.32 \pm 0.89$ & $1.35 \pm 1.16$ & 0.636 \\
\hline Tumor necrosis factor- $\alpha(\mathrm{pg} / \mathrm{mL})$ & $1.92 \pm 1.28$ & $2.71 \pm 1.44$ & 0.015 \\
\hline Interleukin-6 (pg/mL) & $2.45 \pm 3.19$ & $1.38 \pm 1.36$ & 0.113 \\
\hline
\end{tabular}

Table 2

Echocardiographic and biochemical data of study patients after surgery

\begin{tabular}{lccc}
\hline & Atrial fibrillation group $(n=56)$ & Sinus control group $(n=32)$ & $p$ value \\
\hline Left atrial diameter $(\mathrm{mm})$ & $43.5 \pm 9.6$ & $35.0 \pm 5.5$ & $<0.001$ \\
Left atrial area $\left(\mathrm{cm}^{2}\right)$ & $29.8 \pm 12.0$ & $21.0 \pm 7.0$ & $<0.001$ \\
Right atrial area $\left(\mathrm{cm}^{2}\right)$ & $20.0 \pm 6.4$ & $16.3 \pm 5.1$ & 0.009 \\
Left ventricular ejection fraction $(\%)$ & $65.0 \pm 10.8$ & $65.5 \pm 12.4$ & 0.800 \\
high-sensitivity C-reactive protein $(\mathrm{mg} / \mathrm{L})$ & $2.01 \pm 1.44$ & $1.71 \pm 1.45$ & 0.222 \\
Tumor necrosis factor- $\alpha(\mathrm{pg} / \mathrm{mL})$ & $3.27 \pm 4.05$ & $3.03 \pm 2.04$ & 0.745 \\
Interleukin-6 $(\mathrm{pg} / \mathrm{mL})$ & $1.66 \pm 1.32$ & $1.44 \pm 1.39$ & 0.323 \\
\hline
\end{tabular}

\subsection{Factors associated with sinus conversion by the radiofrequency maze procedure in $A F$ patients}

The mean duration of follow-up in the AF group was $55.0 \pm 17.5$ months (range, 12 to 84 months). Two deaths occurred during follow-up: one sudden death at 12-month follow-up and one death from hepatic encephalopathy at 18 -month follow-up. Sinus con- version after radiofrequency maze procedure was defined as achieving persistent sinus rhythm without antiarrhythmic drug therapy or electrical cardioversion at 2-month follow-up and thereafter. Thirty-seven patients achieved persistent sinus conversion following the radiofrequency maze procedure (sinus conversion group), and 19 patients did not regain sinus rhythm (sinus non-conversion group). 
Table 3

Baseline clinical characteristics and biochemical data of atrial fibrillation patients with and without sinus conversion after maze procedure

\begin{tabular}{|c|c|c|c|}
\hline & Sinus converters $(n=37)$ & Sinus non-converters $(n=19)$ & $p$ value \\
\hline Age (years) & $54 \pm 13$ & $58 \pm 14$ & 0.328 \\
\hline Men (\%) & $19(51.4 \%)$ & $7(36.8 \%)$ & 0.303 \\
\hline Body mass index $\left(\mathrm{kg} / \mathrm{m}^{2}\right)$ & $24.1 \pm 4.1$ & $23.9 \pm 2.77$ & 0.856 \\
\hline Tricuspid regurgitation (\%) & $25(67.6 \%)$ & $14(73.7 \%)$ & 0.637 \\
\hline Aortic valve disease $(\%)$ & $5(13.5 \%)$ & $2(10.5 \%)$ & 1.000 \\
\hline Hyperlipidemia (\%) & $6(16.2 \%)$ & $5(26.3 \%)$ & 0.368 \\
\hline Coronary artery disease & $3(8.1 \%)$ & $0(0 \%)$ & 0.544 \\
\hline WBC (cells/uL) & $6446.0 \pm 1889.8$ & $6205.3 \pm 2303.3$ & 0.406 \\
\hline Preoperative left atrial diameter (mm) & $51.9 \pm 9.5$ & $59.3 \pm 10.2$ & 0.028 \\
\hline Preoperative left atrial area $\left(\mathrm{cm}^{2}\right)$ & $40.2 \pm 15.4$ & $56.9 \pm 21.9$ & 0.002 \\
\hline Preoperative right atrial area $\left(\mathrm{cm}^{2}\right)$ & $23.2 \pm 6.7$ & $32.1 \pm 9.0$ & 0.001 \\
\hline Postoperative left atrial diameter (mm) & $39.7 \pm 6.9$ & $50.8 \pm 9.9$ & $<0.001$ \\
\hline Postoperative left atrial area $\left(\mathrm{cm}^{2}\right)$ & $24.8 \pm 6.3$ & $39.6 \pm 14.5$ & $<0.001$ \\
\hline Postoperative right atrial area $\left(\mathrm{cm}^{2}\right)$ & $17.2 \pm 3.7$ & $25.6 \pm 6.8$ & $<0.001$ \\
\hline Postoperative left ventricular ejection fraction (\%) & $66.5 \pm 11.0$ & $62.1 \pm 10.1$ & 0.090 \\
\hline Postoperative high-sensitivity C-reactive protein (mg/L) & $2.06 \pm 1.60$ & $1.88 \pm 0.99$ & 0.966 \\
\hline Postoperative tumor necrosis factor- $\alpha$ (pg/mL) & $3.26 \pm 4.73$ & $3.30 \pm 2.32$ & 0.306 \\
\hline Postoperative interleukin-6 (pg/mL) & $1.48 \pm 1.04$ & $2.05 \pm 1.75$ & 0.336 \\
\hline
\end{tabular}

Table 4

Multiple stepwise logistic regression analysis of baseline characteristics and biochemical data of atrial fibrillation patients in predicting atrial fibrillation after maze procedure

\begin{tabular}{lccc}
\hline Variables & Odds ratio & $95 \%$ CI & $p$ value \\
\hline Duration of atrial fibrillation (months) & 1.030 & $1.010-1.051$ & 0.003 \\
Left atrial area $\left(\mathrm{cm}^{2}\right)$ & 1.062 & $1.012-1.115$ & 0.014 \\
Right atrial area $\left(\mathrm{cm}^{2}\right)$ & 1.153 & $1.011-1.314$ & 0.034 \\
high-sensitivity C-reactive protein (mg/L) & 2.623 & $0.846-8.131$ & 0.095 \\
\hline
\end{tabular}

$\mathrm{CI}=$ confidence interval.

Table 3 lists the baseline clinical characteristics for AF patients with and without sinus conversion after maze procedure. The preoperative level of hs-CRP was significantly lower in the sinus conversion group than in the sinus non-conversion group. However, the sinus conversion and sinus non-conversion groups did not significantly differ in preoperative levels of IL-6 and TNF- $\alpha$ and postoperative levels of hs-CRP, IL- 6 and TNF- $\alpha$. The preoperative and postoperative left atrial area, left atrial diameter and right atrial area were significantly smaller in the sinus conversion group than the sinus non-conversion group. The pre-surgery duration of AF was significantly shorter in the sinus conversion group than in the sinus non-conversion group. In multivariate analysis, preoperative hs-CRP level was no longer a predictor for persistent AF after surgery. After adjustments for preoperative hs-CRP level, the only significant variable other than AF duration and preoper- ative left and right atrial sizes in univariate analysis, via multiple stepwise logistic regression analysis (Table 4) independent factors associated with sinus conversion by radiofrequency maze procedure were identified as AF duration, with an odds ratio for persistent AF after surgery of 1.030 for each 1-month increment in AF duration (95\% confidence interval 1.010 to $1.051, p=$ 0.003 ), and preoperative left atrial area, with an odds ratio for persistent $\mathrm{AF}$ after surgery of 1.062 for each $1-\mathrm{cm}^{2}$ increment in preoperative left atrial area $(95 \%$ confidence interval 1.012 to $1.115, p=0.014$ ).

\subsection{Preoperative duration of $A F$ and left atrial size as factors associated with the success of sinus conversion by the radiofrequency maze procedure in AF patients}

Discriminant analysis was performed to identify the duration of $\mathrm{AF}$ as factor associated with $\mathrm{AF}$ develop- 


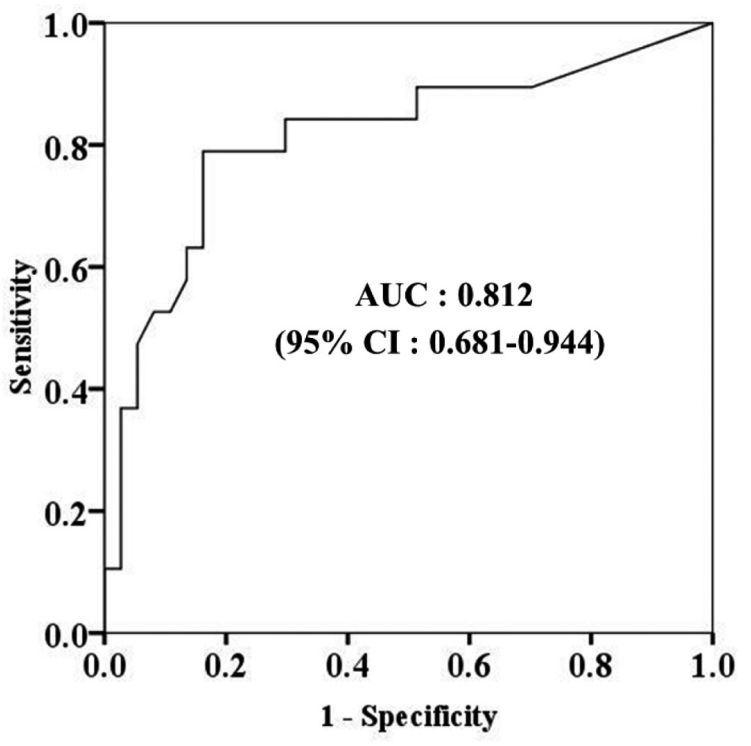

Fig. 1. Receiver Operator Characteristic curve for the cut-off value of the duration of AF in predicting sinus rhythm restoration by the radiofrequency maze procedure. The area under curve (AUC) for the cut-off value of the AF duration of 43.5 months was 0.812 [95\% confidence interval (CI), $(0.681,0.944), p<0.001]$. The sensitivity and specificity of the cut-off value of 43.5 months were $78.9 \%$ and $83.8 \%$, respectively.

ment after the radiofrequency maze procedure. The area under the Receiver Operator Characteristic curve for the cut-off value of 43.5 months was 0.812 (95\% confidence interval, [0.681, 0.944]) (Fig. 1). The sinus conversion rate was significantly lower in patients with $\mathrm{AF}$ duration $>43.5$ months than in patients with AF duration $<43.5$ months (odds ratio 14.47, 95\% confidence interval 3.77 to $55.48, p<0.001$ ).

Discriminant analysis was performed to identify the preoperative left atrial area as factor associated with AF development after radiofrequency maze procedure. The area under the Receiver Operator Characteristic curve for the cut-off value of $37.5 \mathrm{~cm}^{2}$ was $0.760(95 \%$ confidence interval, [0.636, 0.883]) (Fig. 2). The sinus conversion rate was significantly lower in patients with preoperative left atrial area $>37.5 \mathrm{~cm}^{2}$ than in patients with left atrial area $<37.5 \mathrm{~cm}^{2}$ (odds ratio 21.18 , $95 \%$ confidence interval 2.56 to $175.53, p=0.002$ ). None of the patients with preoperative left atrial area $<33.5 \mathrm{~cm}^{2}$ developed AF after radiofrequency maze procedure. Additionally, the area under the Receiver Operator Characteristic curve for the cut-off value of the preoperative left atrial diameter of $51.5 \mathrm{~mm}$ was 0.681 (95\% confidence interval, $[0.530,0.831])$. The sinus conversion rate was lower in patients with preoperative left atrial diameter $>51.5 \mathrm{~mm}$ than in patients

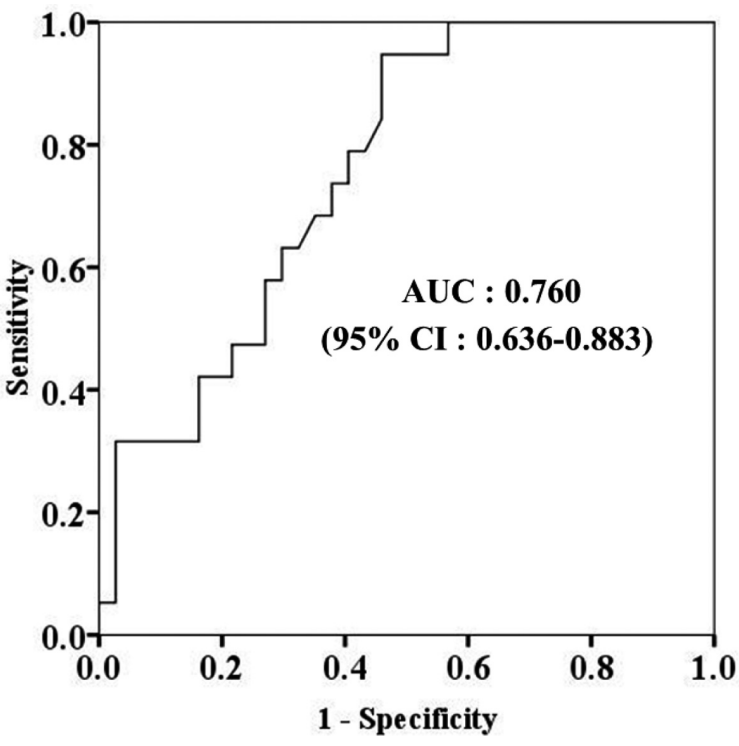

Fig. 2. Receiver Operator Characteristic curve for the cut-off value of the preoperative left atrial area in predicting sinus rhythm restoration by the radiofrequency maze procedure. The area under curve (AUC) for the cut-off value of the preoperative left atrial area of $37.5 \mathrm{~cm}^{2}$ was 0.760 [95\% confidence interval (CI), $(0.636,0.883), p=0.002$ ]. The sensitivity and specificity of the cut-off value of $37.5 \mathrm{~cm}^{2}$ were $94.7 \%$ and $54.0 \%$, respectively.

with left atrial area $<51.5 \mathrm{~mm}$ (odds ratio $2.857,95 \%$ confidence interval [0.794, 10.280], $p=0.101$ ).

For the entire AF group, AF event-free survival was $100 \%$ for patients with preoperative AF duration $\leqslant 43.5$ months and preoperative left atrial area $\leqslant$ $37.5 \mathrm{~cm}^{2}, 72.2 \%$ for patients with preoperative AF duration $\leqslant 43.5$ months and preoperative left atrial area $\geqslant 37.5 \mathrm{~cm}^{2}$, and $23.5 \%$ for patients with preoperative AF duration $\geqslant 43.5$ months and preoperative left atrial area $\geqslant 37.5 \mathrm{~cm}^{2}$ (Fig. 3). The incidence of AF during follow-up was significantly greater in patients with preoperative AF duration $\geqslant 43.5$ months and preoperative left atrial area $\geqslant 37.5 \mathrm{~cm}^{2}$ than in those with preoperative AF duration $\leqslant 43.5$ months and preoperative left atrial area $\leqslant 37.5 \mathrm{~cm}^{2}(p<0.001)$. The incidence of AF during follow-up was significantly greater in patients with preoperative $\mathrm{AF}$ duration $\leqslant 43.5$ months and preoperative left atrial area $\geqslant 37.5 \mathrm{~cm}^{2}$ than in those with preoperative AF duration $\leqslant 43.5$ months and preoperative left atrial area $\leqslant 37.5 \mathrm{~cm}^{2}(p=0.017)$.

\section{Discussion}

This study examined the clinical and biochemical factors associated with sinus conversion by the radiofre- 


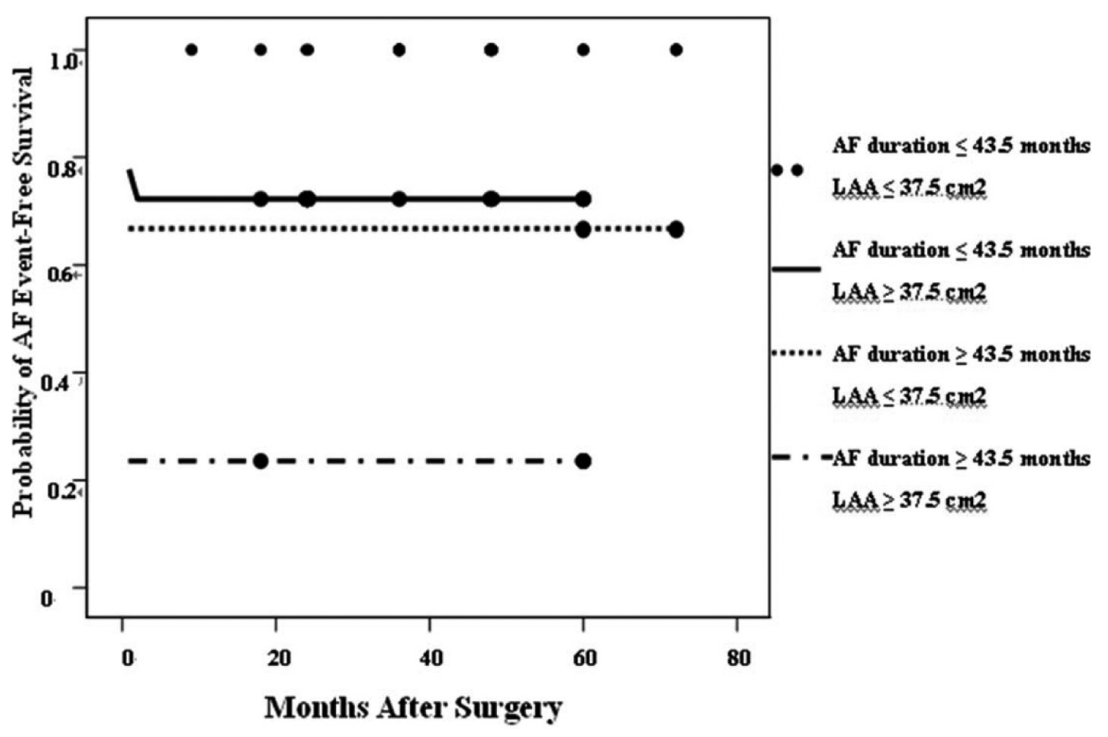

Fig. 3. Atrial fibrillation (AF) event-free survival curves following radiofrequency maze procedure among AF patients that have been divided into four groups based on whether they were above or below the threshold of the preoperative AF duration and left atrial area (LAA). Notably, the incidence of AF was significantly higher in patients with preoperative AF duration $>43.5$ months and LAA $>37.5 \mathrm{~cm}^{2}$ than in patients with preoperative AF duration $<43.5$ months and LAA $<37.5 \mathrm{~cm}^{2}(p<0.001)$.

quency maze procedure for patients with mitral valve disease and persistent AF. This study demonstrated that plasma inflammatory markers, hs-CRP, IL-6 and TNF$\alpha$, were not significant factors associated with sinus non-conversion by the radiofrequency maze procedure for AF. Preoperative AF duration and preoperative left atrial size were identified as an independent factors associated with sinus conversion by the radiofrequency maze procedure.

Epidemiological and clinical studies have shown an association between CRP and risk of developing future AF [8]. However, CRP, an acute-phase protein and a marker of systemic inflammation, is produced in nonspecific physiological and biochemical responses to most forms of organ or tissue damage, infection and inflammation [9] Additionally, Marcus et al. showed that there was a transcardiac gradient of CRP [15]. Thus, plasma inflammatory levels may not accurately reflect the genuine inflammatory status of atrial myocardium $[15,16]$. This study showed that plasma inflammatory markers, hs-CRP, IL- 6 and TNF- $\alpha$, were not important factors associated with sinus non-conversion by the radiofrequency maze procedure for AF. Thus, this study showed that plasma inflammatory markers were not significant risk factors for failed maze procedures. This finding suggests that a systemic anti-inflammatory strategy may not be appropriate for therapeutic intervention after radiofrequency maze procedure adjunct to mitral valve surgery. Our finding is supported by the clinical observation that the routine use of statins, angiotensin converting enzyme inhibitor or type I angiotensin II receptor blocker do not improve outcomes of AF catheter ablation [17].

Atrial fibrillation is the most frequent sustained cardiac arrhythmia in patients with mitral valve disease, contributing to increased risk of mortality and systemic embolization [2,18]. Additionally, development of AF after cardiac surgery is associated with increased morbidity and mortality [19]. The saline-irrigated radiofrequency maze procedure successfully restores sinus rhythm and atrial contractility in patients with persistent AF and mitral valve disease [6,20,21] and improves survival, late cardiac function, and freedom from late stroke [22]. We have shown that atrial size reduction after radiofrequency maze procedure adjunct to mitral valve surgery predicts the success of sinus conversion after surgery [6]. Matsuo et al. have recently shown that continuous AF duration predicts maintenance of sinus rhythm after catheter ablation for persistent AF [23]. Longer duration of persistent AF and large left atrium indicated more structural remodeling of the atria.

\subsection{Study limitations}

Only 12-lead surface electrocardiograms had been used in the follow-up period. This may be a limited way of rhythm monitoring. 


\section{Conclusion}

Plasma inflammatory markers are not associated with sinus non-conversion by the radiofrequency maze procedure adjunct to mitral valve surgery.

\section{Acknowledgements}

We would like to thank the Kaohsiung Chang Gung Memorial Hospital, Chang Gung University College of Medicine, Taiwan for financially supporting this research under Contract number (CMRPG860391).

\section{References}

[1] Chesebro JH, Fuster V and Halperin JL, Atrial fibrillation-risk marker for stroke, $N$ Engl J Med 323 (1990), 1556-1558.

[2] Benjamin EJ, Wolf PA, D'Agostino RB, Silbershatz H, Kannel $\mathrm{WB}$ and Levy D, Impact of atrial fibrillation on the risk of death: The Framingham heart study, Circulation 98 (1998), 946-952.

[3] Patel JB, Borgeson DD, Barnes ME, Rihal CS, Daly RC and Redfield MM, Mitral regurgitation in patients with advanced systolic heart failure, J Card Fail 10 (2004), 285-291.

[4] Trichon BH, Felker GM, Shaw LK, Cabell CH and O'Connor $\mathrm{CM}$, Relation of frequency and severity of mitral regurgitation to survival among patients with left ventricular systolic dysfunction and heart failure, Am J Cardiol 91 (2003), 538-543.

[5] Wilson PW, An epidermiologic perspective of systemic hypertension, ischemic heart disease, and heart failure, Am J Cardiol 80 (1997), 3J-8J.

[6] Chen MC, Chang JP, Guo GBF and Chang HW Atrial size reduction as a predictor of the success of radiofrequency maze procedure for chronic atrial fibrillation in patients undergoing concomitant valvular surgery, J Cardiovasc Electrophysiol 12 (2001), 867-874.

[7] Chen MC, Chang JP, Chang HW, Chen CJ, Yang CH, Chen $\mathrm{YH}$ and $\mathrm{Fu} \mathrm{M}$, Clinical determinants of sinus conversion by radiofrequency maze procedure for persistent atrial fibrillation in patients undergoing concomitant mitral valvular surgery, Am J Cardiol 96 (2005), 1553-1557.

[8] Aviles RJ, Martin DO, Apperson-Hansen C, Houghtaling PL, Rautaharju P, Kronmal RA, Tracy RP, Van Wagoner DR, Psaty $\mathrm{BM}$, Lauer MS and Chung MK, Inflammation as a risk factor for atrial fibrillation, Circulation 108 (2003), 3006-3010.

[9] Montenero AS, Mollichelli N, Zumbo F, Antonelli A, Dolci A, Barberis M, Sirolla C, Staine T, Fiocca L, Bruno N and O'Connor S, Helicobacter pylori and atrial fibrillation: A possible pathogenic link, Heart 91 (2005), 960-961.

[10] Roldán V, Marín F, Blann AD, García A, Marco P, Sogorb F and Lip GY, Interleukin-6, endothelial activation and thrombogenesis in chronic atrial fibrillation, Eur Heart J 24 (2003), 1373-1380.

[11] Akar JG, Jeske W and Wilber DJ, Acute onset human atrial fibrillation is associated with local cardiac platelet activation and endothelial dysfunction, J Am Coll Cardiol 51 (2008), 1790-1793.

[12] Schnabel RB, Larson MG, Yamamoto JF, Sullivan LM, Pencina MJ, Meigs JB, Tofler GH, Selhub J, Jacques PF, Wolf PA, Magnani JW, Ellinor PT, Wang TJ, Levy D, Vasan RS and Benjamin EJ, Relations of biomarkers of distinct pathophysiological pathways and atrial fibrillation incidence in the community, Circulation 121 (2010), 200-207.

[13] Chung MK, Martin DO, Sprecher D, Wazni O, Kanderian A, Carnes CA, Bauer JA, Tchou PJ, Niebauer MJ, Natale A and Van Wagoner DR, C-reactive protein elevation in patients with atrial arrhythmias: Inflammatory mechanisms and persistence of atrial fibrillation, Circulation 104 (2001), 2886-2891.

[14] Chen MC, Chang JP, Chen CJ, Yang CH, Hung WC, Fu M and Yeh $\mathrm{KH}$, Atrial pacemaker complex preserved radiofrequency maze procedure reducing the incidence of sick sinus syndrome in patients with atrial fibrillation, Chest 128 (2005), 25712575 .

[15] Marcus GM, Smith LM, Ordovas K, Scheinman MM, Kim AM, Badhwar N, Lee RJ, Tseng ZH, Lee BK and Olgin JE, Intracardiac and extracardiac markers of inflammation during atrial fibrillation, Heart Rhythm 7 (2010), 149-154.

[16] Chen MC, Chang JP, Liu WH, Yang CH, Chen YL, Tsai TH, Wang YH and Pan KL, Increased inflammatory cell infiltration in the atrial myocardium of patients with atrial fibrillation, $\mathrm{Am}$ J Cardiol 102 (2008), 861-865.

[17] Richter B, Derntl M, Marx M, Lercher P and Gössinger HD, Therapy with angiotensin-converting enzyme inhibitors, angiotensin II receptor blockers, and statins: no effect on ablation outcome after ablation of atrial fibrillation, Am Heart $J$ 153 (2007), 113-119.

[18] Grigioni F, Avierinos JF, Ling LH, Scott CG, Bailey KR, Tajik AJ, Frye RL and Enriquez-Sarano M, Atrial fibrillation complicating the course of degenerative mitral regurgitation: determinants and long-term outcome, J Am Coll Cardiol 40 (2002), 84-92.

[19] Almassi GH, Schowalter T, Nicolosi AC, Aggarwal A, Moritz TE, Henderson WG, Tarazi R, Shroyer AL, Sethi GK, Grover FL and Hammermeister KE, Atrial fibrillation after cardiac surgery: a major morbid event? Ann Surg 226 (1997), 501511.

[20] Chen MC, Guo BF, Chang JP, Yeh KH and Fu M, Radiofrequency and cryoablation of atrial fibrillation in patients undergoing valvular operations, Ann Thorac Surg 65 (1998), 16661672 .

[21] Sie HT, Beukema WP, Elvan A and Ramdat Misier AR, Longterm results of irrigated radiofrequency modified maze procedure in 200 patients with concomitant cardiac surgery: six years experience, Ann Thorac Surg 77 (2004), 512-516.

[22] Bando K, Kasegawa H, Okada Y, Kobayashi J, Kada A, Shimokawa T, Nasu M, Nakatani S, Niwaya K, Tagusari O, Nakajima H, Hirata M, Yagihara T and Kitamura S, Impact of preoperative and postoperative atrial fibrillation on outcome after mitral valvuloplasty for nonischemic mitral regurgitation, J Thorac Cardiovasc Surg 129 (2005), 1032-1040.

[23] Matsuo S, Lellouche N, Wright M, Bevilacqua M, Knecht S, Nault I, Lim KT, Arantes L, O'Neill MD, Platonov PG, Carlson J, Sacher F, Hocini M, Jaïs P and Haïssaguerre M, Clinical predictors of termination and clinical outcome of catheter ablation for persistent atrial fibrillation, J Am Coll Cardiol 54 (2009), 788-795. 


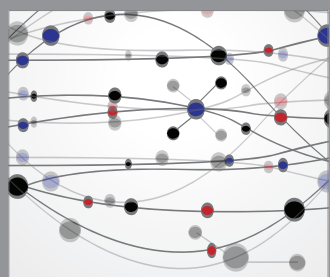

The Scientific World Journal
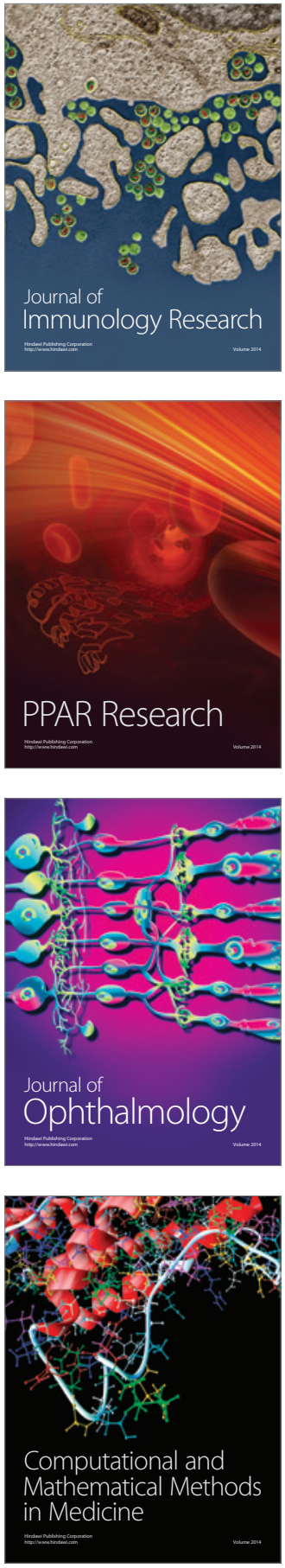

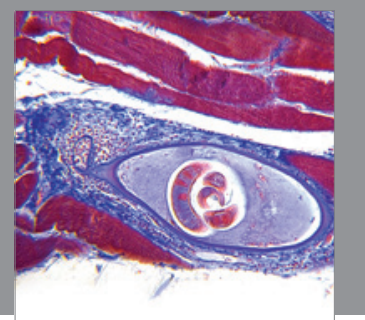

Gastroenterology

Research and Practice
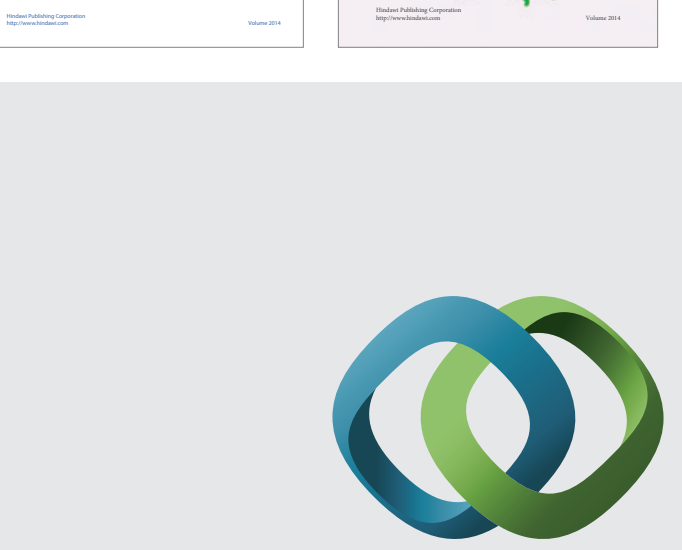

\section{Hindawi}

Submit your manuscripts at

http://www.hindawi.com
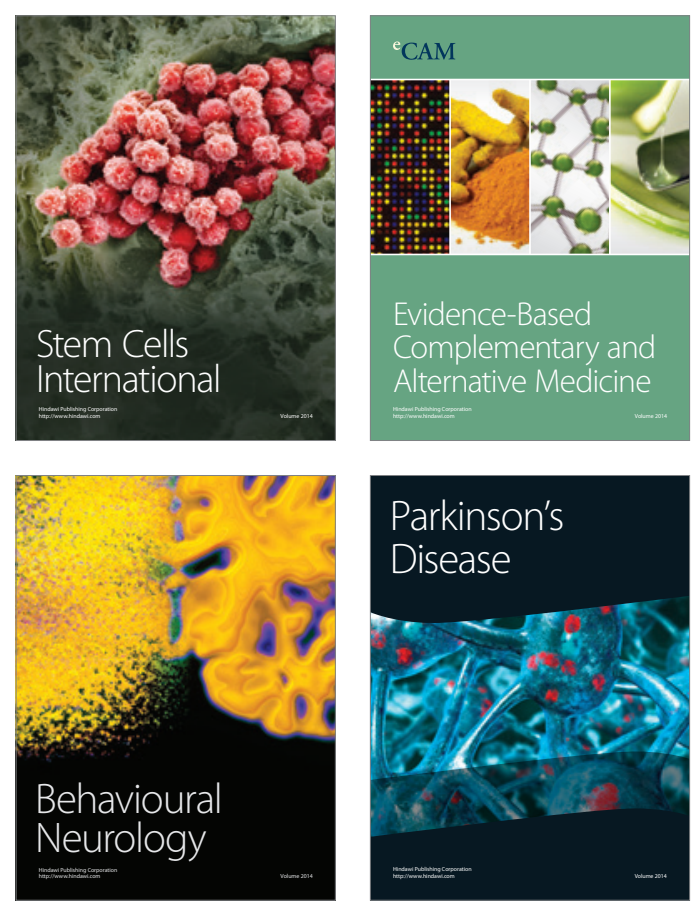

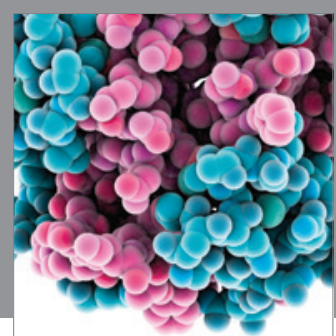

Journal of
Diabetes Research

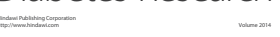

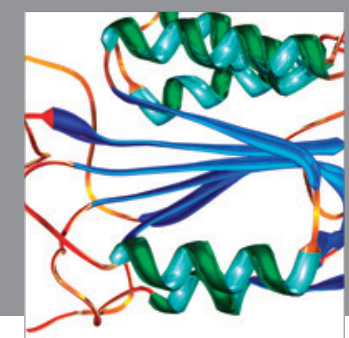

Disease Markers
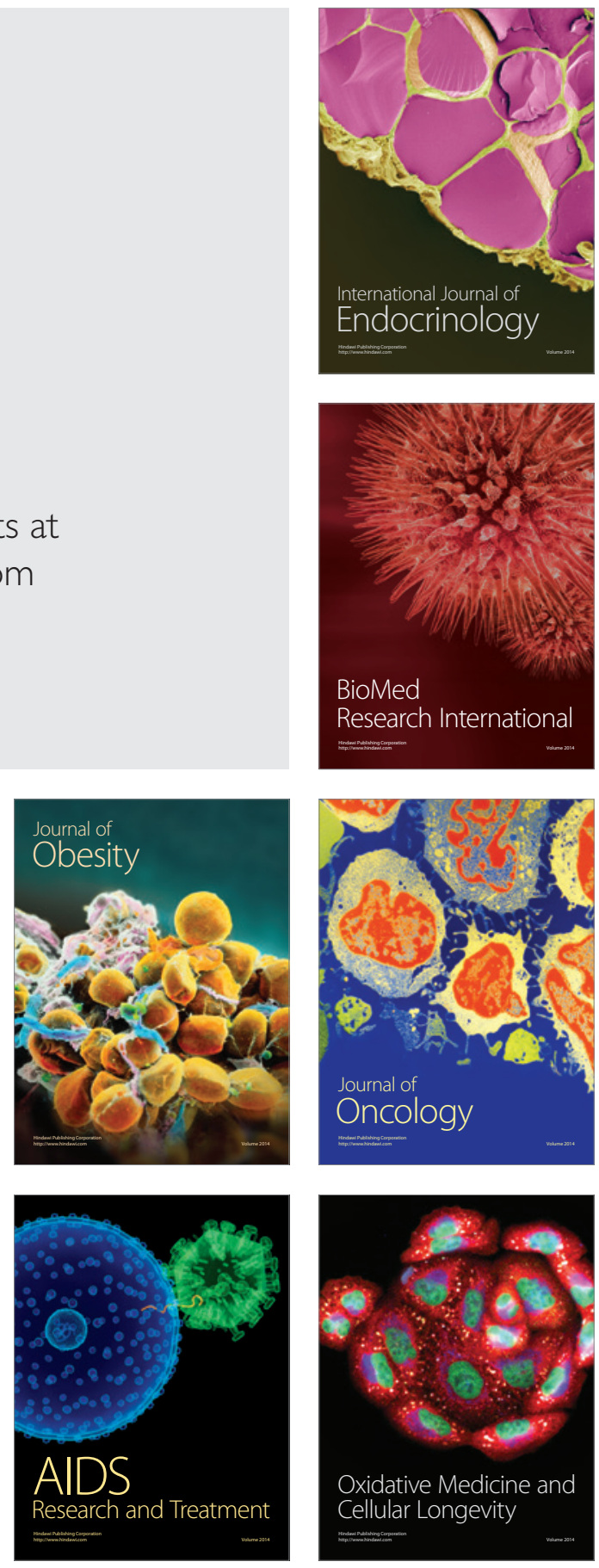